\title{
Rapid and Inexpensive Evaluation of Nonstandard Amino Acid Incorporation in Escherichia coli
}

Jordan W. Monk, Sean P. Leonard, Colin W. Brown, Michael J. Hammerling, Catherine Mortensen, Alejandro E. Gutierrez, Nathan Y. Shin, Ella Watkins, Dennis M. Mishler*, Jeffrey E. Barrick*

Center for Systems and Synthetic Biology, Department of Molecular Biosciences, The University of Texas at Austin, Austin, Texas 78712, United States

*E-mail: dennis.mishler@gmail.com, jbarrick@cm.utexas.edu 
a
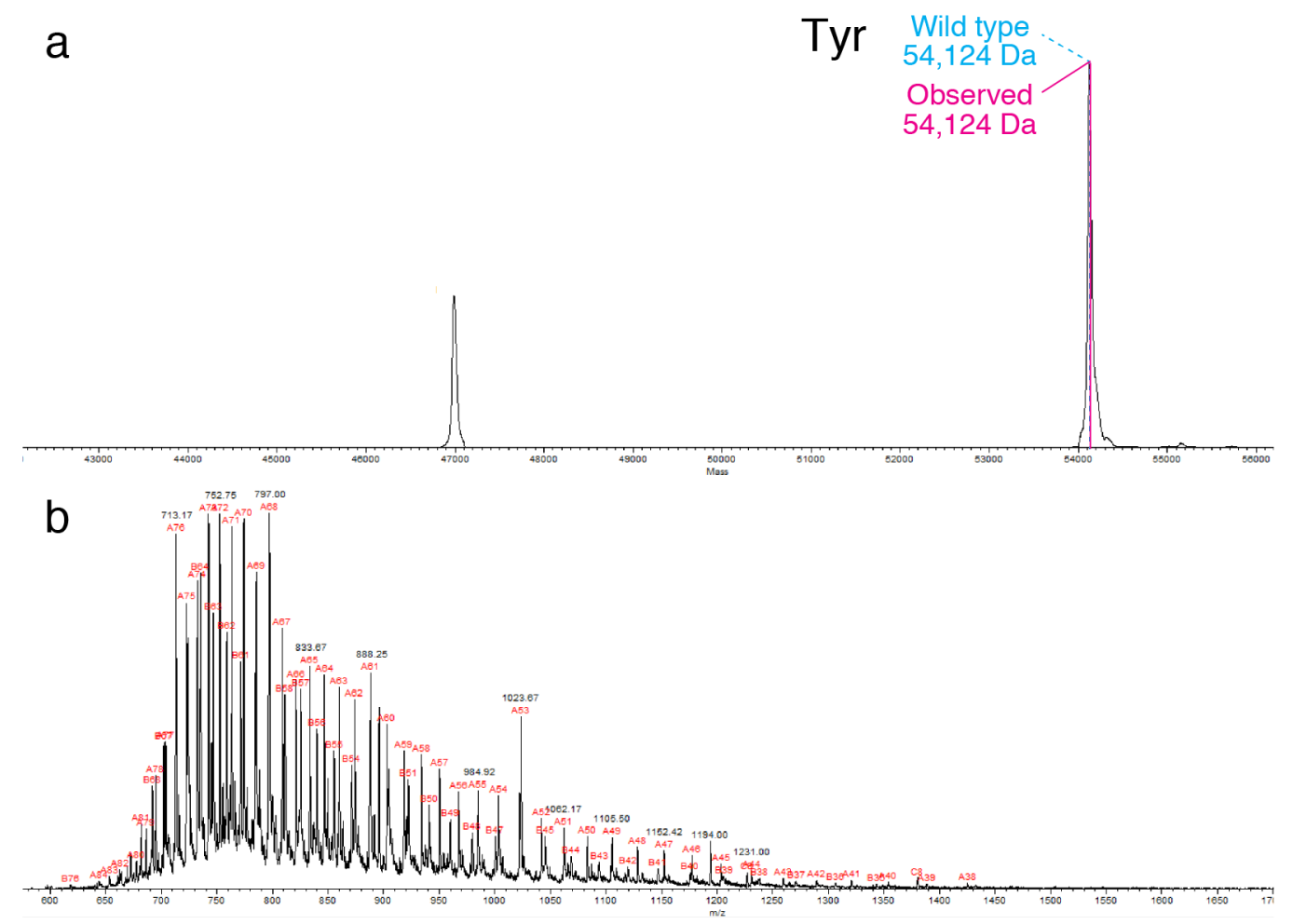

Figure S1. Fidelity of the Tyr OTS evaluated by mass spectrometry. Deconvoluted (a) and raw $\mathrm{m} / \mathrm{z}$ (b) electrospray ionization mass spectrometry (ESI-MS) spectra of the intact mRFP1-sfGFP fusion expressed from the assay plasmid (pRXG) in C321. $\mathrm{A}$.exp cells containing the Tyr OTS plasmid after purification via a C-terminal $\mathrm{His}_{6}$ tag. The wild type mass expected for tyrosine incorporation at the TAG codon in the linker (cyan) and the experimentally observed MS peak (magenta) are labeled on the deconvoluted plot. In this case, the observed peak corresponds to tyrosine incorporation at TAG, as expected. 

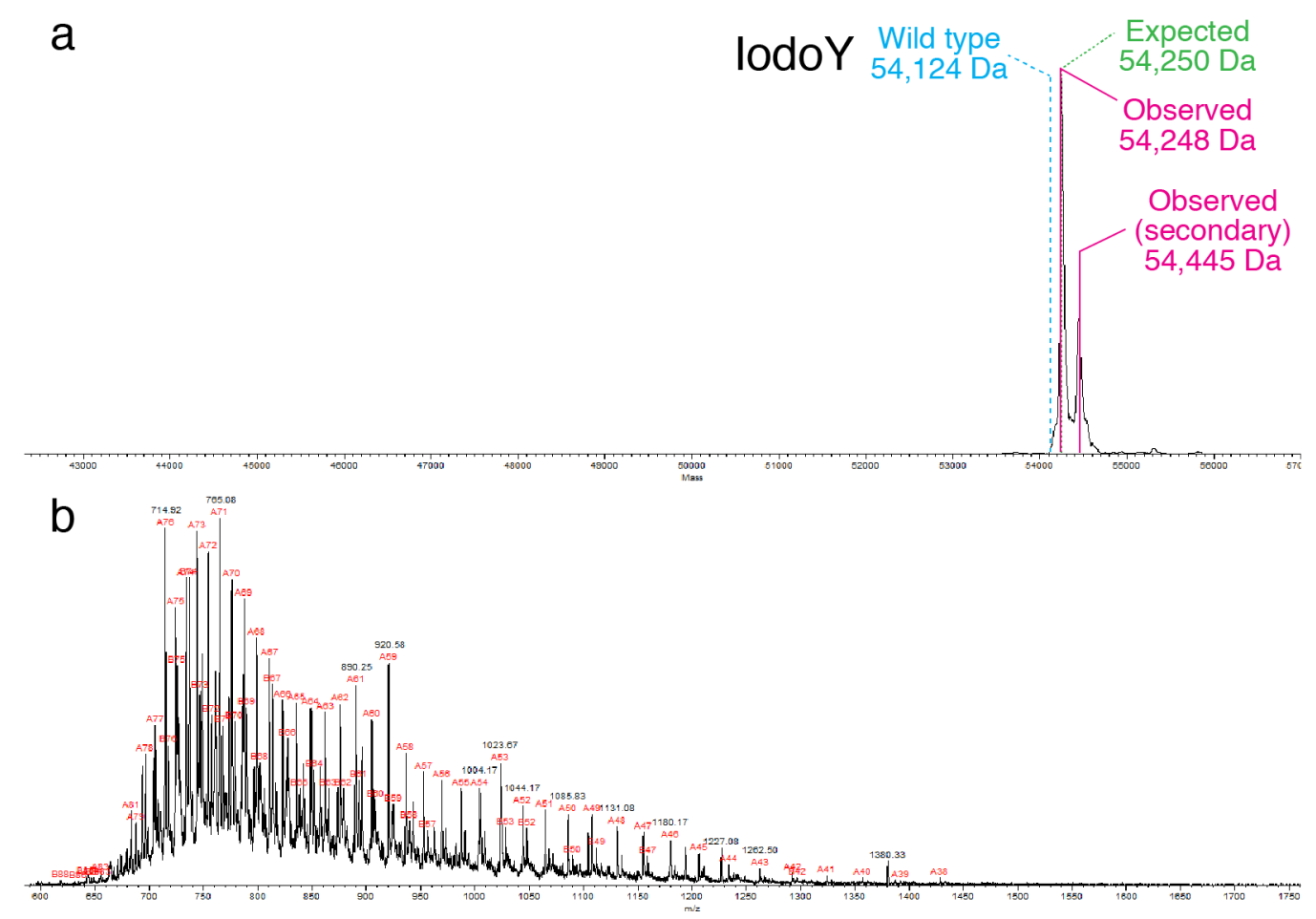

Figure S2. Fidelity of the IodoY OTS evaluated by mass spectrometry. Deconvoluted (a) and raw $\mathrm{m} / \mathrm{z}$ (b) electrospray ionization mass spectrometry (ESI-MS) spectra of the intact mRFP1sfGFP fusion expressed from the assay plasmid (pRXG) in C321. $\Delta$ A.exp cells containing the IodoY OTS plasmid after purification via a $\mathrm{C}$-terminal $\mathrm{His}_{6}$ tag. The wild type mass expected for tyrosine incorporation at the TAG codon in the linker (cyan), expected mass for 3-iodotyrosine incorporation at the TAG codon in the linker (green), and experimentally observed MS peaks (magenta) are labeled on the deconvoluted plot. The major peak observed at 54,248 Da corresponds to incorporation of 3-iodotyrosine. No peak is found for the wild-type mass expected for tyrosine incorporation, indicating very high OTS fidelity. The minor secondary peak at 54,445 Da may correspond to oxidative damage or another protein modification. 

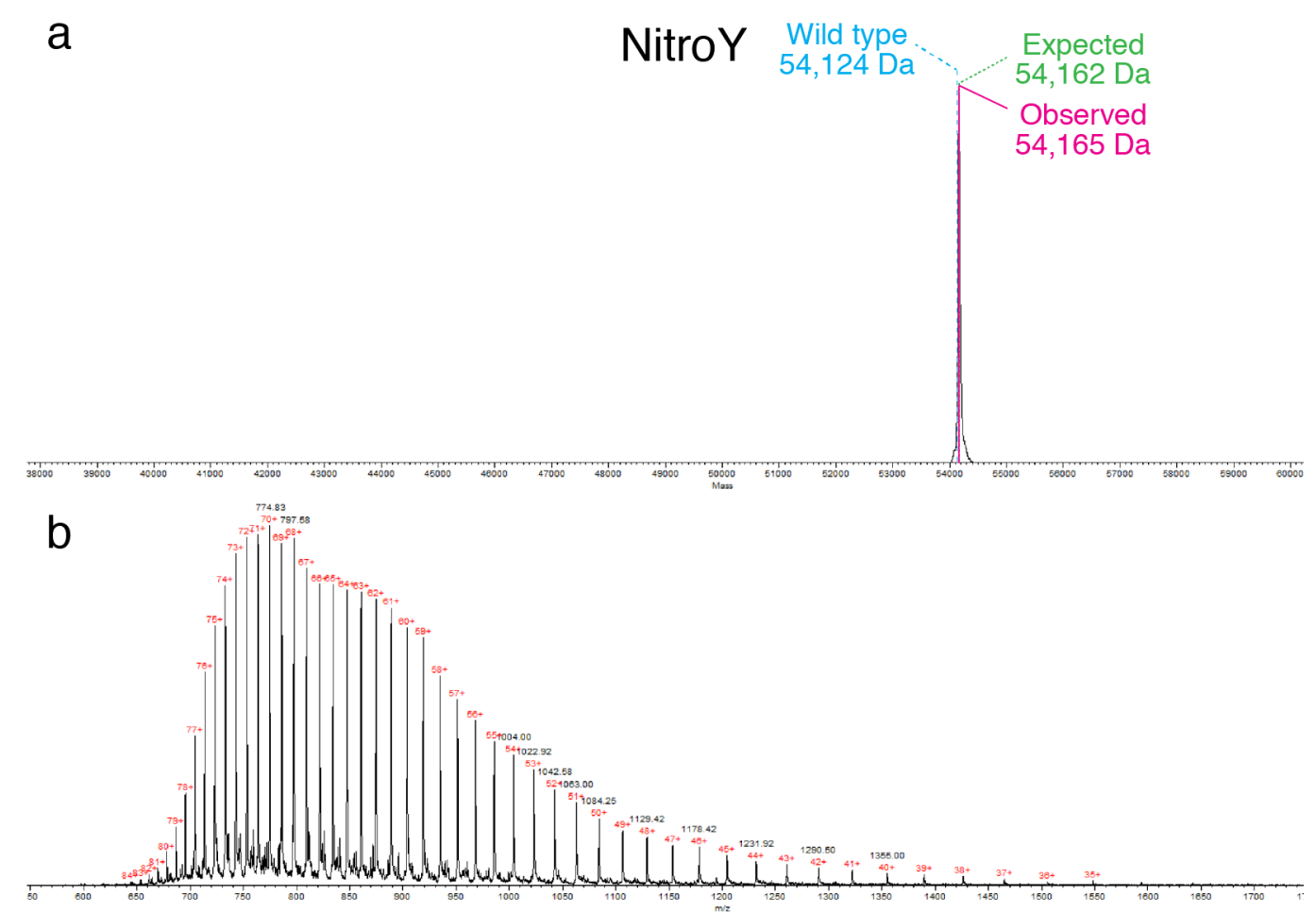

Figure S3. Fidelity of the NitroY OTS evaluated by mass spectrometry. Deconvoluted (a) and raw $\mathrm{m} / \mathrm{z}$ (b) electrospray ionization mass spectrometry (ESI-MS) spectra of the intact mRFP1sfGFP fusion expressed from the assay plasmid (pRXG) in C321. $\Delta$ A.exp cells containing the NitroY OTS plasmid after purification via a C-terminal His ${ }_{6}$ tag. The wild type mass expected for tyrosine incorporation at the TAG codon in the linker (cyan), expected mass for 3nitrotyrosine incorporation at the TAG codon in the linker (green), and experimentally observed MS peak (magenta) are labeled on the deconvoluted plot. The major peak observed at 54,165 Da corresponds to incorporation of 3-nitrotyrosine. No peak is found for the wild-type mass expected for tyrosine incorporation, indicating very high OTS fidelity. 


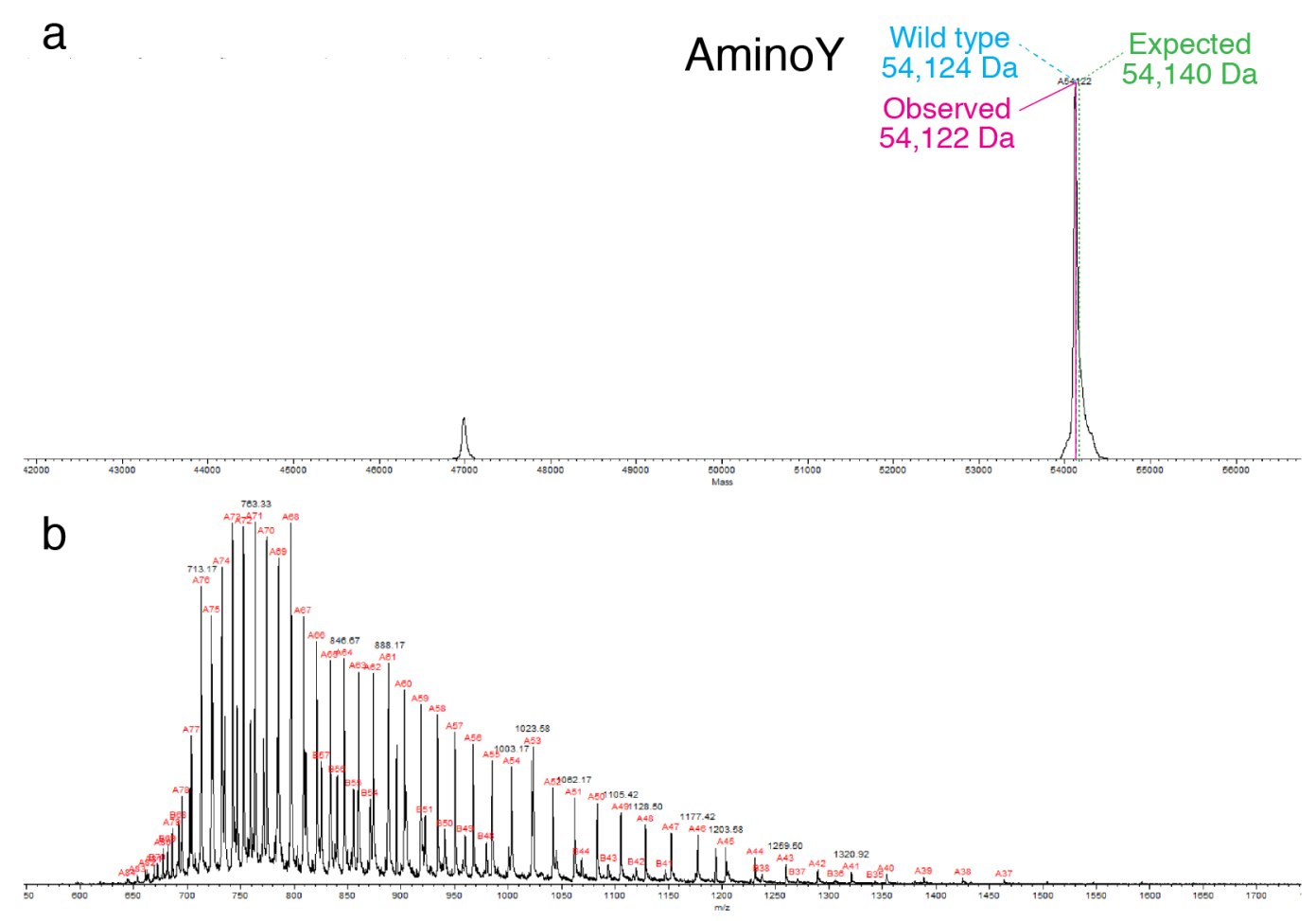

Figure S4. Fidelity of the AminoY OTS evaluated by mass spectrometry. Deconvoluted (a) and raw $\mathrm{m} / \mathrm{z}$ (b) electrospray ionization mass spectrometry (ESI-MS) spectra of the intact mRFP1sfGFP fusion expressed from the assay plasmid ( $\mathrm{pRXG}$ ) in $\mathrm{C} 321 . \Delta \mathrm{A}$.exp cells containing the AminoY OTS plasmid after purification via a C-terminal $\mathrm{His}_{6}$ tag. The wild type mass expected for tyrosine incorporation at the TAG codon in the linker (cyan), expected mass for 3aminotyrosine incorporation at the TAG codon in the linker (green), and experimentally observed MS peak (magenta) are labeled on the deconvoluted plot. The major peak observed at 54,122 Da corresponds most closely to incorporation of tyrosine. A minor, unresolved side-peak overlapping the main mass peak that corresponds to incorporation of 3-aminotyrosine at TAG may also be present, but overall these results indicate that this OTS has a very low fidelity. 

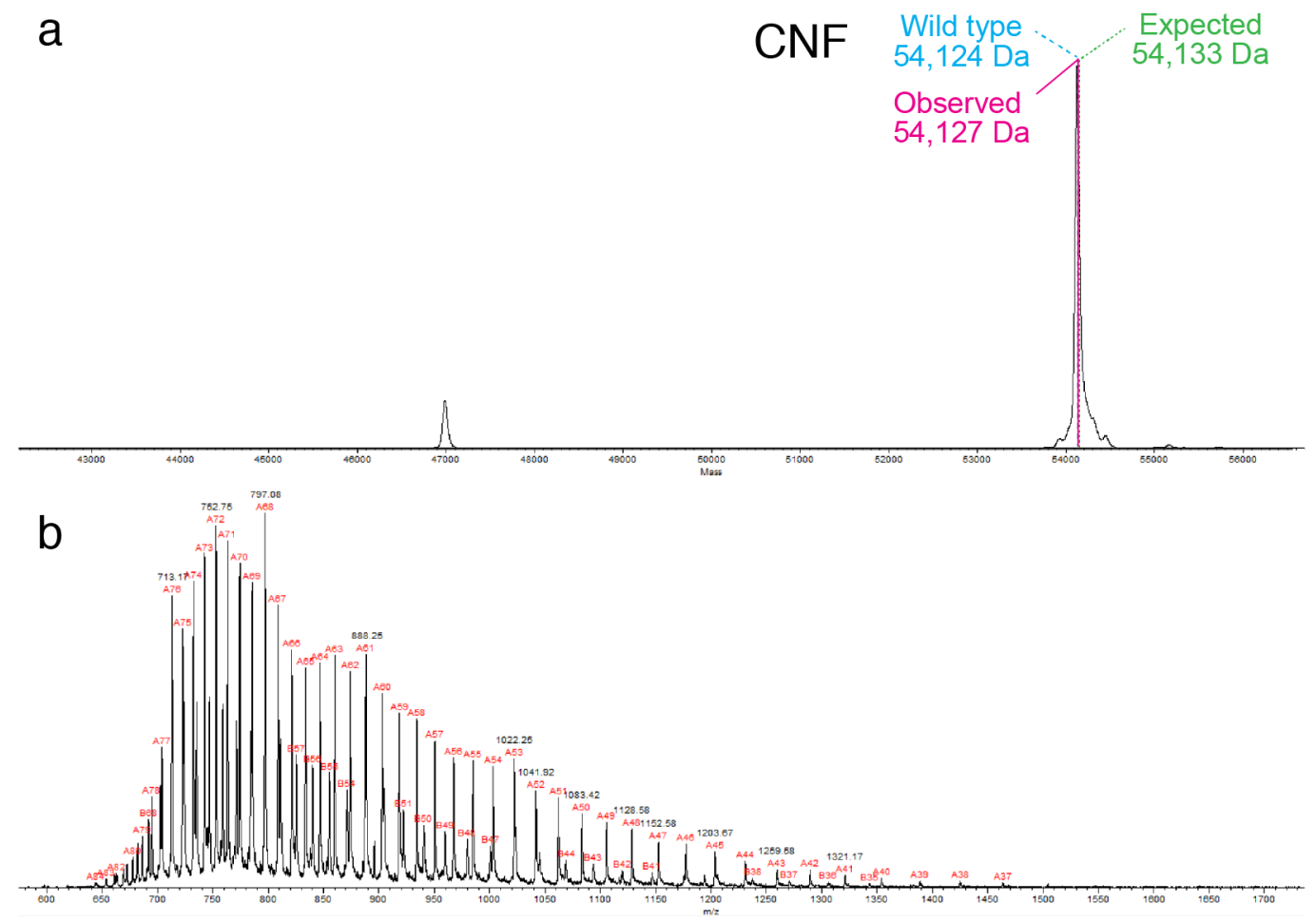

Figure S5. Fidelity of the CNF OTS evaluated by mass spectrometry. Deconvoluted (a) and raw $\mathrm{m} / \mathrm{z}$ (b) electrospray ionization mass spectrometry (ESI-MS) spectra of the intact mRFP1-sfGFP fusion expressed from the assay plasmid (pRXG) in C321. $\Delta$ A.exp cells containing the CNF OTS plasmid after purification via a $\mathrm{C}$-terminal $\mathrm{His}_{6}$ tag. The wild type mass expected for tyrosine incorporation at the TAG codon in the linker (cyan), expected mass for 4-cyanophenylalanine incorporation at the TAG codon in the linker (green), and experimentally observed MS peak (magenta) are labeled on the deconvoluted plot. The major peak observed at 54,127 Da corresponds most closely to incorporation of tyrosine. A minor, unresolved side-peak overlapping the main mass peak that corresponds to incorporation of 4-cyanophenylalanine at TAG may also be present, but overall these results indicate that this OTS has a very low fidelity. 